\title{
IQ consortium perspective: complementary LBA and LC-MS in protein therapeutics bioanalysis and biotransformation assessment
}

\author{
Surinder Kaur*,1, Kevin P Bateman² (D) Jim Glick³ ${ }^{3}$, Mark Jairaj ${ }^{4}$, John F Kellie ${ }^{5}$, Jens Sydor ${ }^{5}$ \\ \& Jianing Zeng ${ }^{6}$ \\ ${ }^{1}$ Department of BioAnalytical Sciences, Genentech, a member of the Roche Group, South San Francisco, CA 94080, USA \\ 2PPDM, Merck \& Co., Inc., West Point, PA 19486, USA \\ ${ }^{3}$ PK Sciences, Bioanalytics, Novartis Institutes for BioMedical Research, Novartis, East Hanover, NJ 07936, USA \\ ${ }^{4}$ Translational Biomarkers \& Bioanalysis, UCB, Slough, UK \\ ${ }^{5}$ In Vitro/In Vivo Translation, GSK, Collegeville, PA 19426, USA \\ ${ }^{6}$ Bioanalytical Sciences, Translational Medicine Department, BMS, Princeton, NJ 08540, USA \\ *Author for correspondence: kaur.surinder@gene.com
}

Increasingly diverse large molecule modalities have driven the need for complex bioanalysis and biotransformation assessment involving both traditional ligand-binding assays (LBA) and more recent hybrid immunoaffinity LC-MS platforms. Given the scientific expertise in LBA and LC-MS typically resides in different functions within the industry, this has presented operational challenges for an integrated approach for bioanalysis and biotransformation assessment. Encouragingly, over time, the industry has recognized the complementary value of the two platforms. This has not been an easy transition as organizational structures vary widely within the industry. However, there are tremendous benefits in adopting fully integrated strategies for biopharma. This IQ consortium paper presents current perspectives across the biopharma industry. It highlights the technical and operational challenges in current large molecule bioanalysis, the value of collaborations across LBA and LC-MS, and scientific expertise for fully integrated strategies for bioanalysis and biotransformation.

First draft submitted: 19 November 2019; Accepted for publication: 3 February 2020; Published online:

25 February 2020

Keywords: biotransformation $\bullet$ hybrid ligand binding LC-MS • immunoaffinity $\bullet$ LC-MS $\bullet$ LC-MS/MS $\bullet$ MS $\bullet$ peptides $\bullet$ Proteins

In recent years, MS-based approaches like hybrid immunoaffinity (IA) LC-MS have been established as an alternative to traditional ligand-binding assays (LBA) for the bioanalysis of biotherapeutics [1,2]. This use has been driven largely by increasingly complex large molecular modalities in the biopharmaceutical industry pipelines, for example, therapeutic proteins, monoclonal antibodies (mAbs), bispecific antibodies, antibody drug conjugates and fusion proteins. The growing complexity of new biotherapeutics was propelled both by advancements in protein engineering capabilities and the desire to reach previously considered less 'druggable' targets. New modalities present challenges for bioanalysis during drug discovery and development, which are best resolved by an expanded and integrated bioanalytical strategy including both LBA and IA LC-MS analytical platforms that are essentially a hybrid of LBA and LC-MS. For historical reasons, LBA and LC-MS platforms are often placed in different functional areas, making a seamless co-ordination of analytical approaches for a fully integrated strategy operationally challenging.

Traditionally, therapeutic proteins have been most commonly quantified by LBA due to their high sensitivity, amenability for high-throughput analyses and the lack of alternative technologies available in the past. Although LBAs are widely available and have been the industry standard for nonclinical and clinical pharmacokinetics (PK) assessment, they exhibit a critical dependence on the availability of high-quality reagents for both capture and detection [3]. Moreover, their performance may be negatively impacted if the reagents available do not achieve the 
necessary selectivity and affinity. Additionally, the growing complexity of biotherapeutics requires in vivo structural characterization of biotherapeutics for biotransformation assessment, whereas LBA provide binding data.

In the past, LC-MS assays have been employed as the workhorse for the bioanalysis of small molecule analytes. These assays are robust, exhibit wide linear dynamic ranges and can be rapidly developed to simultaneously analyze multiple analytes. More recently, protein LC-MS, particularly when linked to IA enrichment, has emerged as an attractive platform for sensitive and selective protein biomarkers and protein therapeutic quantification. These advantages highlight the utility of LC-MS as a viable and attractive platform for protein bioanalysis, complementary to LBA despite some challenges in achieving the highest sensitivity. Affinity enrichment strategies are commonly used to increase sensitivity by removing interfering matrix components in plasma or tissue matrices. Furthermore, assay variability can be corrected by using an internal standard (IS) like an isotopically labeled peptide, a flanged peptide (with a tryptic cleavage site) or a full-length protein.

Across the industry, no consensus regarding a biotherapeutics bioanalytical strategy has yet been achieved about when and how to implement LC-MS methods across the pipeline. To date, different organizations have been implementing the LC-MS platform at various stages of drug discovery and development, based on their individual technical and operational factors. Overall, a close collaboration across LBA and LC-MS experts in an organization will help address how to integrate the LC-MS platform in a more strategic manner for increasingly complex large molecule pipeline challenges. In addition, different approaches are taken by these organizations while outsourcing protein LC-MS assays based on specific challenges, such as the availability of contract research organizations (CROs) with the required expertise, equipment and sufficient capacity of CROs. There is no single regulatory guidance that addresses the validation of proteolytic digestion-based or hybrid LBA/LC-MS methods for therapeutic proteins, including the recent ICH M10 draft guidance. A recent industry white paper has recommended that a combination of existing guidelines for chromatography assays (used for small molecules) and LBA assays (used for large molecules) is appropriate for the validation of hybrid LBA/LC-MS methods [4].

To address the paucity of an industry consensus regarding approaches to implement LC-MS protein bioanalysis and provide general guidance for implementing an integrated large molecule analytical strategy incorporating varying analytical platforms, a Working Group for Large Molecule LC-MS Analysis comprising representatives from approximately 20 biopharmaceutical companies was formed under the umbrella of the International Consortium for Innovation and Quality in Pharmaceutical Development (IQ Consortium). The remit of the IQ Working Group was to specifically discuss and disseminate in presentations and publications, the best use of LC-MS for biotherapeutic analysis and biotransformation. In 2017, the IQ Working Group organized a joint IQ/AAPS Workshop in San Diego, "LBA and LC-MS: Why incorporate both for large molecule drug bioanalysis?" with 11 speakers, including a US FDA speaker. The day long workshop had over 50 attendees and received positive feedback on the scientific content and the collaborative LBA/LC-MS atmosphere.

This white paper aims to provide general guidance and a consensus view of the participating companies with respect to LC-MS for biotherapeutics bioanalysis implementation. The scope for biotherapeutic quantification by LC-MS is limited to approaches involving peptide analysis; intact protein analysis workflows are not covered herein as they are less widely used at present. Key characteristics of LBA, LC-MS, hybrid approaches and operational considerations will be discussed; PK and biotransformation case studies will be reviewed; considerations for outsourcing of biotherapeutics LC-MS assays and the regulatory landscape will be discussed.

\section{Key characteristics of LBA, LC-MS \& hybrid IA LC-MS/MS \& operational considerations}

Initially, when LC-MS technology emerged as an alternative approach for the bioanalysis of biotherapeutics, the proponents of the technology focused on the technical advantages of LC-MS compared with LBA. It is acknowledged that this may have caused untoward tension within the large molecule bioanalytical community. Fortunately, this was transient, as the scientific community recognized the value of utilizing multiple platforms in a complementary manner to move programs forward. Quite often, the scientists with expertise in LC-MS and LBA sit in different functions or laboratories within the organization. As we move forward with this new paradigm, we recognize that cross-functional training and collaboration are some of the most important aspects to operationalize the wider adoption of LC-MS and hybrid IA LC-MS for the bioanalysis of biotherapeutics.

In general, for high-dose nonclinical or clinical studies, where $C_{\text {trough }}$ concentrations are in the $\mu \mathrm{g} / \mathrm{ml}$ range, sensitivity is not an issue, and LBA, LC-MS or IA LC-MS can provide quantitative data. In this case, the strategy may be driven by the availability of suitable reagents, requirement of a generic or specific assay format and operational considerations such as program timelines (Table $1 \&$ Figure 1). At the discovery stage, for example, 


\begin{tabular}{|c|c|c|c|c|}
\hline Platform & Reagents & Sensitivity/identity & Selectivity/multiplexing & Training/outsourcing \\
\hline LBA & $\begin{array}{l}\text { - One or two high-quality } \\
\text { binding reagents for } \\
\text { performance }\end{array}$ & $\begin{array}{l}\text { - Microfluidic platforms for } \\
\mathrm{pg} / \mathrm{ml} \text { sensitivity }\end{array}$ & $\begin{array}{l}\text { - Binding specificity } \\
\text { - Total or free format } \\
\text { - Potential for interference } \\
\text { - Sample dilution typical } \\
\text { - Multiplexed possible }\end{array}$ & $\begin{array}{l}\text { - Established analysts ready CRO } \\
\text { capacity } \\
\text { - Low capital investment } \\
\text { - Assay maintenance } \\
\text { requirements }\end{array}$ \\
\hline $\begin{array}{l}\text { Direct Digest } \\
\text { LC-MS/MS }\end{array}$ & $\begin{array}{l}\text { - No binding reagents required } \\
\text { - Stable-labeled peptide/protein }\end{array}$ & $\begin{array}{l}\text { - Limited sensitivity }(1 \mu \mathrm{g} / \mathrm{ml} \text { for } \\
\mathrm{mAb}) \\
\text { - Biotransformation ID }\end{array}$ & $\begin{array}{l}\text { - Specificity based on: } \\
\text { (i) retention time, } \\
\text { (ii) peptide mass, (iii) peptide } \\
\text { fragmentation } \\
\text { - Potential for interference } \\
\text { - No dilution (except } C_{\max } \text { ) } \\
\text { - Total format } \\
\text { - Highly multiplexed }\end{array}$ & $\begin{array}{l}\text { - Few expert CROs } \\
\text { - Building CRO capacity is a slow } \\
\text { process } \\
\text { - Initial capital investment } \\
\text { - Robust assay transfer }\end{array}$ \\
\hline $\begin{array}{l}\text { Hybrid } \\
\text { IA-LC-MS/MS }\end{array}$ & $\begin{array}{l}\text { - One binding reagent used to } \\
\text { enrich analyte } \\
\text { - Stable-labeled peptide/protein }\end{array}$ & $\begin{array}{l}\text { - Sensitivity }(\mathrm{pg} / \mathrm{ml}) \text { with } \\
\text { antipeptide reagents } \\
\text { - Biotransformation ID }\end{array}$ & $\begin{array}{l}\text { - Specificity based on: } \\
\text { (i) binding, (ii) retention time, } \\
\text { (iii) peptide mass, (iv) peptide } \\
\text { fragmentation } \\
\text { - Total or free format } \\
\text { - Low potential for interference } \\
\text { - No dilution (except } C_{\text {max }} \text { ) } \\
\text { - Total format } \\
\text { - Highly multiplexed }\end{array}$ & $\begin{array}{l}\text { - Limited industry expertise } \\
\text { - Few expert CROs } \\
\text { - Building CRO capacity is a slow } \\
\text { process } \\
\text { - Initial capital investment } \\
\text { - Robust assay transfer }\end{array}$ \\
\hline
\end{tabular}

$C_{\text {max }}$ : Maximum concentration; CRO: Contract research organization; IA-LC-MS/MS: Immunoaffinity LC-MS; ID: Identification; LBA: Ligand-binding assay; mAb: Monoclonal antibody.

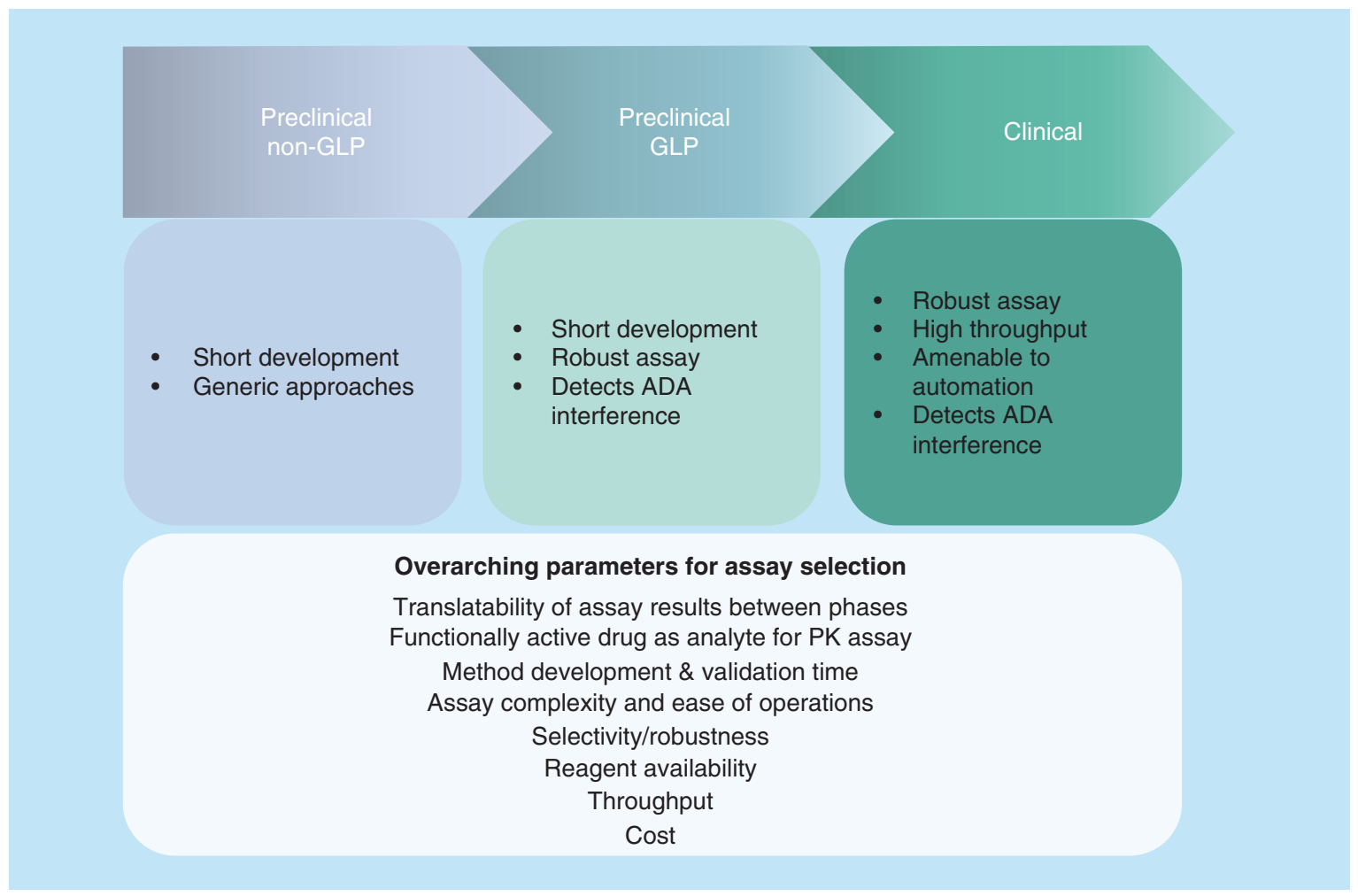

Figure 1. Operational considerations for assay strategy during the drug-development process.

ADA: Antidrug antibody; GLP: Good laboratory practice; PK: Pharmacokinetics.

LC-MS-based assays for human mAbs can be developed rapidly using a generic antihuman Fc antibody capture reagent in the absence of a specific capture reagent [5]. However, in some cases it may be advantageous to use orthogonal approaches to answer specific questions, for example if the binding specificity of reagents is in question, orthogonal direct digestion LC-MS can be used to confirm that reagents are capturing the analyte appropriately. 
When $\mathrm{C}_{\text {trough }}$ concentrations are in the $\mathrm{ng} / \mathrm{ml}$ range, direct digestion LC-MS will not provide sufficient sensitivity and the availability of assay reagents becomes more important. With appropriate reagents, either LBA or hybrid IA LC-MS can be used. Although for the IA LC-MS assay, the capture reagent is used for enrichment only and the reagent quality is less critical, it is still important to apply LBA expertise to optimize the binding step [6]. It is important to recognize that only the analytes captured during the IA step in the assay will be detected by LC-MS.

Another consideration may be the need for simultaneous (multiplexed) measurements in studies that involve dosing drug combinations. IA LC-MS can be readily multiplexed to quantify multiple biotherapeutics simultaneously using surrogate peptides from each protein [7]. Ultra-high sensitivity in the sub-ng/ml range is challenging for both LBA and IA LC-MS, and the reagent requirement becomes critical for both approaches. Sensitivity in this range has not typically been required for nonclinical or clinical PK assessment in the past, and was more common for biomarker analysis. However, new drug modalities or drug delivery methods may require highly sensitive assays for PK assessment. For example, antibody drug conjugates and cancer immunology bispecific antibodies require low doses due to safety concerns, or their respective therapeutic window. Ophthalmology biotherapeutics with intravitreal dosing, or inhaled biotherapeutics, result in low systemic concentrations. Recent microfluidic LBA platforms enable sub-ng/ml sensitivity and high-throughput analysis, and require high-quality capture and detection reagents, however, assay validation experience on some new platforms is limited at this time.

The IA LC-MS approach for sub-ng/ml sensitivity involves elaborate sample preparation and treatment such as sequential immunocapture of the intact analyte followed by the peptide analyte [8], microflow- or nano-flow LC, 2D LC [5], and multiple-mechanism enrichment [9]. Further, sub-ng/ml sensitivity typically involves generating additional reagents, such as antipeptide antibodies, and results in lower sample throughput.

All these considerations for the different assay platforms are important when choosing an assay at different stages in the drug discovery and development process where varying sensitivity, robustness and timelines may be required. Ultimately, there are two key areas of focus: the scientific question to be addressed with the assay data and the operational considerations during assay execution.

While addressing the scientific question, we should recognize that biotherapeutic proteins, in contrast to small molecules, often have microheterogeneity. Proteins can exist in different isoforms and can have a variety of posttranslational modifications within specification limits established during the manufacturing process development. The large molecular size further complicates the assay strategy as, unlike small molecules, neither LBA nor peptidebased LC-MS assay formats measure the entire molecule. In the former, the capture and detection relies on two binding epitopes, and in the latter, it relies on one or two peptides and one binding epitope (as in the case of IA LC-MS). In addition, proteins may be tightly bound to a soluble target or form other complexes, such as antidrug antibody (ADA) complexes. In this case, addressing the scientific question may require the measurement of the 'free' unbound form or the 'total' bound and unbound concentration. The assay conditions, such as sample dilution or fractionation may impact the amount of 'free' or 'total' measured. Moreover, proteins can also be complex mixtures where LBA reagents may not bind to all the components in the mixture, and MS-based assays can provide additional information due to their selectivity and multiplexing capabilities. For example, bioanalysis of complex antibody-drug conjugates during discovery and development greatly benefit from the use of LC-MS based methodologies [10]. Both total and conjugated antibodies can be quantified by LC-MS and in contrast to LBA, the exact ratio of payload to antibody can be determined [10-12]. Another key difference in the quantification and characterization of large molecules in biological samples is also the ability of LC-MS-based methods to determine biotransformations of protein therapeutics as well as isomer characterization of biomarkers [6].

Using LBA expertise to optimize protein-protein interactions for highly specific sample isolation combined with protein chemistry and LC-MS expertise for highly specific analyte detection and structural characterization, provides tremendous opportunities to address scientific questions which could not be previously addressed using any single technology. Multidisciplinary collaborations are important for leveraging the diverse methodologies and expertise for state-of-the-art drug discovery and development.

\section{Further considerations for LC-MS \& hybrid IA LC-MS strategies}

Large molecule LC-MS-based workflows for the analysis of protein biotherapeutics can be divided into several classes based on increasing workflow complexity: direct digestion (such as pellet digestion), IA enrichment (either protein or peptide capture) and intact protein methods. Within each of these broad categories, modifications of the general workflow have been introduced. For example, protein precipitation followed by pellet digestion can have a 
subsequent solid phase extraction step after pellet digestion for additional sample clean-up and signature peptide enrichment. IA enrichment of the protein may be followed by additional IA enrichment of the peptide.

The choice of a large molecule LC-MS workflow may vary depending on the anticipated doses used in the study and the sensitivity requirements for quantification, as discussed above. For nonclinical and clinical studies with $\mathrm{C}_{\text {trough }}$ concentrations in the $\mu \mathrm{g} / \mathrm{ml}$ range, direct digestion can be used for a capture reagent-free approach, keeping in mind that the measurement provides a 'total' drug concentration, in other words, the sum of 'free' drug and any drug that may be complexed to circulating target or ADA. The typical application of the direct digestion workflow involves the use of an organic solvent for the protein precipitation step, followed by resuspension of the pellet, reduction and alkylation, and tryptic digestion to yield the signature peptide. When using this workflow, it is important to consider the possibility that biotherapeutic complexes (e.g., drug-target complexes or ADA-drug complexes) or biotherapeutic aggregates formed during the process may not be completely dissociated prior to trypsin digestion. In most instances, the organic solvents used, such as methanol, acetonitrile, isopropanol with trichloroacetic acid, and acetone, would dissociate any drug complexes formed. However, there is the possibility that the drug complex may not be completely dissociated during precipitation. This could occur in cases of mAbs which bind with very high affinity to a target. In this instance, tryptic digestion and release of the signature peptide could be sterically hindered, resulting in incomplete digestion of the $\mathrm{mAb}$ and under quantification. Increased recoveries of drug (>95\%) when using this workflow may require more aggressive dissociation conditions involving urea and heat treatment prior to protein precipitation and careful resuspension of the pellet. Therefore, when selecting the solvent composition for protein precipitation and resuspension, it is important to demonstrate that potential drug complexes or aggregates have been completely dissociated if a total drug assay is required.

For studies where $\mathrm{C}_{\text {trough }}$ concentrations are below the $\mu \mathrm{g} / \mathrm{ml}$ range, for example, in case of low-dose treatment, non-IV dosing regimens (e.g., subcutaneous, topical, intramuscular and intravitreal) or high clearance biotherapeutics, direct digestion or pellet digestion would not provide the required sensitivity. In addition, if data are needed for the quantification of 'free' drug in a study, the more specific and sensitive hybrid IA LC-MS workflow should be considered using either the target itself or a high-affinity anti-idiotypic antibody. As has been reported in the literature, IA LC-MS methods typically have LLOQ in the range of $50-100 \mathrm{ng} / \mathrm{ml}$ and can be further optimized for sensitivity in the single digit $\mathrm{ng} / \mathrm{ml}$ range [13].

The IA LC-MS assay format can be designed to be generic or specific, depending on the choice of capture reagent. For example, a generic format can be used when quantifying a humanized $m A b$ in a preclinical animal study where 'total' concentration is desired to measure on-target and off-target toxicity. In such studies, the IA capture reagent is used to target the $\mathrm{F}_{\mathrm{c}}$ region of the antibody (e.g., Protein A, goat anti-human Fc or mouse anti-human Fc). When IA LC-MS methods are used in clinical assays, a specific assay format is needed due to the possibility of an endogenous protein interference. For clinical assay development, an anti-idiotypic antibody or the target itself are typically used for drug capture, their respective qualities can be less stringent than those required for LBA, since LC-MS provides additional selectivity by virtue of LC retention time, peptide mass and peptide fragmentation. For clinical quantification, a peptide from the complementarity-determining regions (CDRs) for antibodies or regions that contain mutations on endogenous protein sequences of the therapeutic protein may provide the required specificity.

When considering either the generic or specific IA LC-MS assay formats, the stable isotope label (SIL) IS can be either peptide-based or protein-based if the drug has been prepared with SIL amino acids. Peptide and protein-based IS quantification schemes have been reported with both methods having similar precision and accuracy. However, when using a SIL protein as IS, the absolute recovery of the drug in the matrix is more easily characterized as all steps in the workflow are tracked with the stable label protein.

An alternative IA LC-MS format known as Stable Isotope Standards and Capture by Anti-Peptide Antibodies (SISCAPA) [14,15] involves using an IA enrichment step after protein digestion to capture a signature peptide or peptides. Like other LC-MS methods that do not include IA capture of the intact biotherapeutic, SISCAPA provides, a 'total' concentration measurement [16]. In a published case study, an atypical PK and an apparent interference in the LBA were observed and investigated using SISCAPA [16]. The SISCAPA assay captured peptides from the CDR region of the antibody and showed PK profiles equivalent to the LBA method. This confirmed that the atypical PK was due to the biology and not an artifact of the assay methods [16].

One challenge for LC-MS methods that do not involve IA capture of the intact protein, such as direct digestion or SISCAPA formats, is to understand if the signature peptide quantified was actually derived from the protein drug or from a proteolytic fragment in circulation. Both intact protein and proteolytic fragments would yield 
the signature peptide after trypsin digestion, resulting in over quantification of the biotherapeutic concentration. Indirect evidence to support that only intact protein is present in circulation can be provided by the measurement of additional peptides where the ratio of signature peptide to confirmatory peptides can provide supportive data for the protein concentration [4]. A more direct approach to ensure that the signature peptide is derived from only intact protein is to use a sequential capture affinity-based LC-MS/MS workflow where the intact protein is captured in the first step and then the signature peptide is captured after enzymatic digestion. In a recent publication, an initial generic affinity capture step using Protein $A / G$ to capture all intact mAbs was followed by a secondary IA step, after digestion, targeting a CDR peptide from the biotherapeutic [17].

As mentioned above, quantification of intact proteins is outside the scope of this work due to limited adoption at this time. However, it is worth mentioning that the characterization of intact mass of biotherapeutics in vivo is widely used to provide additional insights, such as stability, for complex biotherapeutics. In this case, the resolution of instruments used is a factor for consideration depending on the mass changes involved. For example, in antibodydrug conjugate (ADC) analysis, various ADC variants with individual PK profiles generated through deconjugation, catabolism or diverse disposition properties may create additional complexity that may require higher resolution than triple quads operated at low resolution. In this case, LC-high resolution MS (LC-HRMS) can offer greater resolution with full-scan mode, and has evolved as an indispensable tool to analyze $\mathrm{mAb}$ stability by characterizing changes in drug-to-antibody ratio (DAR) [18].

\section{The need to understand biotransformation}

Although protein biotherapeutics have been approved and widely used clinically for several decades, biotransformation data have only recently started to emerge $[6,19,20]$. This contrasts starkly with small molecule drug development where metabolism data are routinely required during nonclinical and clinical development [21,22]. There are several reasons that may account for this difference. Historically, unlike small molecule drugs, protein biotherapeutics (mostly mAbs), were believed to be relatively stable in vivo. Moreover, unlike xenobiotic drugs, the breakdown and elimination of protein biotherapeutics was considered a natural process and not likely to present a safety concern. On a more practical note, the bioanalytical technology platform available for large molecules, namely LBA, did not provide direct structural biotransformation information. Thus, historically, the low risk of safety related to biotransformation, together with the limitations of the available analytical technology platforms, contributed to the lack of biotransformation data for biotherapeutics. However, more recently, increasingly complex drug modalities are driving the need to understand biotransformation.

For protein biotherapeutics, it is possible that in vivo structural changes in the functional region (e.g., the CDR region of a $\mathrm{mAb}$ ) could result in a loss of binding to the target (Table 2). Structural changes in the CDR may result in reduced signals in the LBA assay, assuming the assay reagents bind specifically to the CDR. Such a biotransformation would under-measure the $\mathrm{mAb}$ concentration resulting in an apparent unusually faster clearance. However, faster clearance may also result from various other mechanisms such as ADA-mediated or soluble target-mediated, thus biotransformation could not be inferred from faster clearance alone. Given that the LBA platform cannot provide direct evidence of in vivo biotransformation, mitigation strategies have included mutating out potential unstable amino acids 'hot spots' identified during in vitro formulation stability studies. A limitation of this approach is that chemical modifications in vitro may not be representative of in vivo biotransformations from biological mechanisms. Understanding biotransformations in vivo is important since structural changes may reduce binding, effectively neutralizing the therapeutic activity.

The need to understand in vivo biotransformations of large molecule biotherapeutics became critical with the advent of ADCs $[10,23,24]$. With the presence of a cytotoxic drug, biotransformations could be a safety concern in addition to the functional neutralization concern discussed above. For example, cleavage of the linker could release cytotoxic species in circulation. ADC development represented an ideal opportunity to integrate classical LBA with MS-based approaches and lead to novel hybrid approaches involving affinity-based capture of large molecule drugs from biological matrices and MS analysis. Integrated bioanalytical approaches have become important not only to help understand the fate of complex ADCs in vivo, but also for reverse translation of the in vivo stability data to produce more structurally stable ADCs.

Integrated bioanalytical approaches involving LBA, MS and hybrid assays have become increasingly common as large molecule drugs have become more complex including fusion proteins, bispecifics, conjugated proteins and proteins containing non-native amino acids (Table 2) [1,2]. In case of fusion proteins, it is critical to understand the in vivo stability of the protein subunits, as unexpected proteolysis could result in a loss of potency and ultimately 


\begin{tabular}{|c|c|c|}
\hline Structure & Molecule & Biotransformation ${ }^{\dagger}$ molecule domain: risk assessment \\
\hline & $\mathrm{mAb} / \mathrm{lgG}$ & $\begin{array}{l}\text { - CDR: Impact on binding to target, potency and internalization } \\
\text { - Fc effector function binding domain: impact on Fc effector function } \\
\text { - Carbohydrates: impact on PK clearance } \\
\text { - General: impact on PK, immunogenicity, safety and efficacy }\end{array}$ \\
\hline & $A D C$ & $\begin{array}{l}\text { - Antibody: see mAb above } \\
\text { - Linker: changes in drug-to-antibody ratio distributions and potency } \\
\text { - Drug: oxidation etc., while conjugated; deconjugation and ADC adduct formation } \\
\text { - General: limpact on PK, immunogenicity, safety and efficacy }\end{array}$ \\
\hline & Fusion protein & $\begin{array}{l}\text { - Binding domain: impact on potency and internalization } \\
\text { - Fusion domain: cleavage resulting in loss of potency } \\
\text { - Carrier domain: impact on PK clearance } \\
\text { - General: impact on PK, immunogenicity, safety and efficacy }\end{array}$ \\
\hline & $\begin{array}{l}\text { Pegylated protein } \\
\text { multimer }\end{array}$ & $\begin{array}{l}\text { - Protein: immunogenicity, impact on safety and efficacy } \\
\text { - Linker: cleavage/loss of protein/covalent adduct formation, safety and efficacy } \\
\text { - PEG: immunogenicity } \\
\text { - General: impact on PK, immunogenicity, safety and efficacy }\end{array}$ \\
\hline & Cyclic peptide & $\begin{array}{l}\text { - Binding domain: impact on potency and internalization } \\
\text { - Disulfide: reduction/disulfide shuffling/adduct formation, safety and efficacy } \\
\text { - General: impact on PK, immunogenicity, safety and efficacy }\end{array}$ \\
\hline
\end{tabular}

${ }^{\dagger}$ Deamidation, isomerization, oxidation, glycation, bond cleavage (fusion domains, disulfides, carbohydrates, chemical linkers, etc), novel structural modifications, covalent adduct formation and tertiary structure distortion.

ADC: Antibody-drug conjugate; CDR: Complementarity-determining region; IgG: Immunoglobulin G; mAb: Monoclonal antibody; PK: Pharmacokinetics.

reduced efficacy [25]. Fusion proteins, such as, human Fc/peptide fusions involve a pharmacologically active polypeptide recombinantly conjugated to the Fc portion of human immunoglobulin. This essentially combines the biological activity of the peptide with the long in vivo half-life associated with mAbs. Fusion protein constructs do not occur naturally, thus their stability can be unpredictable and should be monitored in vivo during drug development. For example, multiple proteolytic locations were identified for a variety of 'peptibody' fusion proteins to identify the most stable construct in rats using an IA LC-MS-based strategy [25].

The biotransformation data for large molecule biotherapeutics can be obtained for the intact molecule, for the reduced sub-units or for the peptides after proteolytic digestion. A general approach involves enriching the analyte from biological matrices, such as serum or plasma, by affinity capture followed by MS analysis. It is important to optimize affinity capture with a variety of binding reagents to ensure that modified forms are not lost during sample preparation due to reduced binding. Biotransformations involving larger mass shifts can be readily detected at the intact mass level (but may not be contributable to a single structural modification), while smaller mass shifts may require sub-unit analysis, depending on the resolution of the instrument and abundance of biotransformed species. Other approaches involve an enzymatic removal of the Fc of an antibody to detect smaller mass changes in the $\mathrm{F}\left(\mathrm{ab}^{\prime}\right) 2$ region [26]. For detailed structural analysis, it is necessary to analyze at the peptide level using tandem MS to fragment peptides containing biotransformed amino acids and determine the site and nature of modification. In some cases, a biotransformation may involve a structural rearrangement and no mass change, for example, glutamic acid to pyroglutamic acid, where the modified peptide can be deduced from a change in retention time during LC-MS/MS [6]. HRMS significantly improves the ability to detect biotransformations. However, small mass changes, such as, a 1Da shift due to deamidation of asparagine to aspartic and/or isoaspartic acid, may not be easily detected even by HRMS due to overlapping isotopic peaks of these peptides. Thus, LC separation of deamidated peptides from intact peptide is usually needed in order to detect deamidation [27]. Appropriate sample storage and handling are important for all biotransformation characterization studies to ensure artifactual modifications are not introduced.

Biotransformation information may add value across many areas in drug development, and information from clinical samples could be used to reserve-translate and improve later generations of biotherapeutics. Currently, the field has predominantly explored preclinical models and translation across species is not understood. Similarly, 
well-characterized in vitro predictive models are lacking. Clinical biotransformation knowledge will be important to develop predictive in vitro models and understand translation across species. New questions that could not be answered historically during large molecule development can now be explored, such as the nature and extent of biotransformation, the impact of biotransformations on binding of the biotherapeutic to the target, impact on the effector function, impact on the PK and whether biotransformations vary with route of administration or from patient-to-patient. Further studies may be required to determine whether biotransformations affect the potency in vitro and potential impacts on safety and efficacy in vivo. In addition, it may be asked whether the in vitro biotransformations are predictive of in vivo, or if the product specifications for in vitro changes are appropriate in the context of in vivo changes. For example, a tight product specification for deamidation during manufacturing may not be warranted if deamidation to the same or greater extent occurs rapidly in vivo. Such information would be valuable for both drug development and manufacturing.

In summary, understanding biotherapeutic biotransformations in vivo provides opportunities for LBA and MS analytical groups across the organization to collaborate and add a breadth of value across drug research and development.

\section{Industry outsourcing considerations \& challenges}

Due to constrained resources, to build flexibility and avoid setting up internal GLP/GCP laboratories, pharmaceutical companies engage with CRO partners to expand their large molecule LC-MS capability and capacity in a fashion that has been well established for small molecules [28,29]. Different strategies have been implemented based on the individual company's capabilities and capacity, as well as the availability of CROs [30]. Most companies have applied a hybrid outsourcing model by taking advantage of both internal scientific expertise and expanded external capacities. The key considerations while outsourcing LC-MS large molecule assays are technical capability, capacity and timelines.

Even though outsourcing has become increasingly mainstream in the industry [31], most pharmaceutical companies maintain an internal LC-MS and LBA group especially for complicated LC-MS large molecule analysis for several reasons: the companies need to ensure that they have the capacity to support their portfolio when flexible CRO resources are not available, and/or not available in a timely manner; they need to maintain expertise internally to scientifically oversee work being done at CROs and CROs tend to lag behind pharmaceutical companies in terms of the latest expertise (e.g., biotransformation, ultrasensitive quantification and juxtaposition of $\mathrm{PK} /$ biotransformation/functional binding loss). Most importantly, in-house LC-MS and LBA expertise is essential to drive innovation.

The general practice of LC-MS large molecule assay outsourcing in concept is not much different from that of outsourcing a small molecule LC-MS assay. Many of the same general challenges apply, for example, transfer of samples across global sites. However, the 'technology and expertise due diligence evaluation' has some unique challenges that are critical to ensure that the technical capability of the CRO aligns with the project needs. This includes instrumentation such as micro-LC or HRMS, expertise and experience with general protein handling, IA techniques, protein digestion and peptide fragmentation methods. Scientific expertise in troubleshooting large molecule LC-MS assays, which are generally more complicated, is critical. Sufficient experience to enable both the initial work and the implementation in clinical studies further down the road is important. For the sponsor, unlike outsourcing small molecule LC-MS assays, some internal experience with the assay or prequalification of the assay in-house is a plus for the successful transfer of large molecule assays to the CROs.

Although CROs are up to speed in many areas of bioanalytical support, especially for small molecules, they currently are catching up in large molecule analysis with LC-MS. The current trend indicates CRO interest to expand their large molecule capabilities rapidly. To date, only a small number of CROs have full capability in all expertise and technologies used for large molecule analysis, such as immunocapture, reagent evaluation, digestion, micro-LC and HRMS. There are many reasons that might contribute to this situation: the absence of sufficient and consistent demand forecast by CROs to invest capital and resources to build the capability; low initial return on investment as compared with small molecules due to the added technical and operational challenges, the need for specialized instrumentation and initial training, all leading initially to higher resource needs; and a limited availability of the talent pool in large molecule LC-MS analysis.

To mitigate potential CRO gaps in technical capability and capacity, good planning is the key for successful outsourcing of large molecule LC-MS bioanalysis. Proactively training or qualifying a few CROs can be a great help for successful study support. Some companies take advantage of the fixed-capacity resource outsourcing model 
at CROs to provide appropriate capacity. With a fixed resource model, the Pharma sponsor may pay for the instrumentation and can train the staff ahead of time. The same trained staff can then be used for many other sponsor programs in the future.

The cost for outsourcing large molecule LC-MS assays is varied, and it is difficult to have direct comparison with those of small molecule assays and LBA. Besides the cost per sample, other hidden costs need to be taken into consideration, such as reagents, equipment, long term maintenance of the assay, reagent management, technical oversight, etc. The cost of outsourcing small molecule assays is usually lower than that of the large molecules LC-MS assays because of less technical complexity, shorter extraction time, shorter analytical run times, less variability due to the use of the IS and no need for critical reagents. However, the overall cost for large molecule LC-MS assays may not necessarily be higher than that of the LBA, depending on the requirements of the reagents, platform used and complexity of the assay.

Ultimately, a good outsourcing strategy should consider the use of in-house expertise and resources to support large molecule LC-MS method development and transfer. This will help reduce CRO method development time, save on method development costs and identify method liabilities before the Tox or clinical programs start. To successfully on-board a large molecule LC-MS CRO, close monitoring by the sponsor on ideally a low-priority project is usually required to help them gain proficiency. Once a CRO is capable of running complex assays and demonstrate that deliverables and timelines are met, they will become more attractive to other sponsors, which in turn raises their experience and allows them to conduct large molecule LC-MS assays with higher precision and more challenging timelines.

\section{Regulatory landscape}

There is no single regulatory guidance directly addressing method development and validation of proteolyticdigestion-based or hybrid LBA coupled with LC-MS/MS detection for biotherapeutic molecules, including the recent ICH M10 draft guidance [32-36]. In addition, neither guidance covers the biotransformation of large molecules. The recently released FDA Bioanalytical Method Validation guidance states, "The purpose of bioanalytical method development is to define the design, operating conditions, limitations and suitability of the method for its intended purpose and to ensure that the method is optimized for validation." The guidance also states, "The recommendations can be modified with justification, depending on the specific type of bioanalytical method. This guidance reflects advances in science and technology related to validating bioanalytical methods." These principles and the use of existing guidelines for small molecule and large molecules have been adopted by researchers developing novel approaches for the quantification of biotherapeutics using LC-MS based approaches $[4,13]$.

The current pharmaceutical industry recommendation, as of 2020, is to adopt aspects of existing guidance documents with some modifications for large molecule LC-MS assays [4,37]. To utilize hybrid technology in regulated applications, users are currently incorporating or adapting scientifically appropriate experiments and criteria from existing regulatory guidelines for conventional chromatographic methods (applicable to small molecules) or LBA (applicable to large molecules). However, no consensus on the number of peptides and transitions per peptide has been determined, and generally a case-by-case basis is recommended. In addition, no acceptance criteria have been established for use of multiple peptides and multiple transitions for quantitating the target protein. Recommended acceptance criteria based on input from several groups experienced in regulated bioanalysis of large molecules have been published (Table 3). This IQ Working Group also endorses the published acceptance criteria (Table 3).

An increasing body of data from hybrid methods may give rise to new validation guidelines as regulators become more familiar with hybrid technologies. Given that these types of assays are using aspects of both chromatographic assays (LC) and LBA, it is likely that specific criteria will need to be defined and established. Acceptance criteria for hybrid assays should not be the same as standard LC-MS assays when immunocapture-based sample preparation is being used. The FDA guidance document provides different requirements and acceptance criteria for LC versus LBA for several aspects of method validation and sample analysis, including calibration curves, quality control, selectivity, specificity, stability, dilution and incurred sample reanalysis. Additionally, the importance of critical reagents is specifically called out as these are especially important for LBA assays and will also impact the performance of hybrid assays.

In some cases, as a program moves from preclinical to clinical development, the assay type and format may change. For example, a generic format may be used initially during preclinical development to measure total drug concentration for understanding on-target and off-target toxicity. Later, during clinical development, a specific format may be used. The assay platform may also change across studies during drug development, for example 
Table 3. Comparison of conventional method validation parameters for protein ligand-binding assay and small molecule LC-MS/MS, with those proposed for protein LC-MS/MS.

\begin{tabular}{|c|c|c|c|}
\hline Parameter & Protein LBA & Small molecule LC-MS/MS & $\begin{array}{l}\text { Protein LC-MS/MS, using a surrogate peptide } \\
\text { (recommended) }\end{array}$ \\
\hline $\begin{array}{l}\text { Calibration curve regression } \\
\text { function }\end{array}$ & $\begin{array}{l}\text { - Nonlinear with four or five } \\
\text { parameter logistic anchor points } \\
\text { may be used }\end{array}$ & $\begin{array}{l}\text { - Linear preferred, nonlinear } \\
\text { with justification }\end{array}$ & $\begin{array}{l}\text { - Linear recommended when possible; nonlinear models } \\
\text { may be acceptable with some affinity-capture methods }\end{array}$ \\
\hline LLOQ (RE, CV) & Within $\pm 25 \%$ & Within $\pm 20 \%$ & Within $\pm 25 \%$ \\
\hline Calibration standards (RE, CV) & $\begin{array}{l}\text { Within } 20 \% \text { (except LLOQ and } \\
\text { ULOQ) }\end{array}$ & Within 15\% (except LLOQ) & Within $20 \%$ (except LLOQ) \\
\hline Accuracy \& precision (RE, CV) & $\begin{array}{l}\text { - Within } 20 \% \text { (LLOQ/ULOQ QCS } \\
\text { within } 25 \% \text { ) } \\
\text { - Min. six runs }\end{array}$ & $\begin{array}{l}\text { - Within } 15 \% \text { (LLOQ QC within } \\
20 \% \text { ) } \\
\text { - Min. three runs }\end{array}$ & $\begin{array}{l}\text { - Within } 20 \% \text { (LLOQ QC within } 25 \% \text { ) } \\
\text { - Min. three runs }\end{array}$ \\
\hline $\begin{array}{l}\text { Dilutional integrity/linearity } \\
\text { parallelism }\end{array}$ & $\begin{array}{l}\text { - RE, CV within } 20 \% \\
\text { - Dilution series CV within } 30 \% \\
\text { using incurred samples }\end{array}$ & $\begin{array}{l}\text { - RE, CV within } 15 \% \\
\text { - NA }\end{array}$ & $\begin{array}{l}\text { - } \mathrm{RE}, \mathrm{CV} \text { within } 20 \% \\
\text { - NA; may be used for troubleshooting affinity capture } \\
\text { methods }\end{array}$ \\
\hline $\begin{array}{l}\text { Selectivity/specificity } \\
\text { Nonspecific matrix-related } \\
\text { interferences } \\
\text { Using individual matrix lots, } \\
\text { analyzed as blanks and fortified } \\
\text { at the LLOQ level } \\
\text { Also evaluate hemolyzed, lipemic } \\
\text { or relevant disease population } \\
\text { samples, as appropriate }\end{array}$ & $\begin{array}{l}\text { - Ten lots } \\
\text { - LLOQ: } \\
\text { Accuracy within } 25 \% \text { for } 80 \% \text { of } \\
\text { fortified lots }\end{array}$ & $\begin{array}{l}- \text { Six lots } \\
\text { Blanks: } \\
<20 \% \text { of LLOQ } \\
<5 \% \text { of IS } \\
\text { - LLOQ: } \\
\text { Accuracy within } 20 \% \text { for } 80 \% \text { of } \\
\text { fortified lots }\end{array}$ & $\begin{array}{l}-6-10 \text { lots } \\
\text { Blanks: } \\
<20 \% \text { of LLOQ } \\
<5 \% \text { of IS } \\
\text { - LLOQ: } \\
\text { Accuracy within } 25 \% \text { for } 80 \% \text { of fortified lots }\end{array}$ \\
\hline $\begin{array}{l}\text { Specific interferences } \\
\text { Using LLOQ (and sometimes } \\
\text { ULOQ for LBAs) QC samples }\end{array}$ & $\begin{array}{l}\text { - Fortified with available } \\
\text { material (ADA, soluble target } \\
\text { and catabolites) or concomitant } \\
\text { drugs (large molecule) } \\
\text { - Accuracy within } 25 \%\end{array}$ & $\begin{array}{l}\text { - Fortified with available } \\
\text { metabolites or concomitant } \\
\text { drugs, as appropriate } \\
\text { - Accuracy within } 20 \%\end{array}$ & $\begin{array}{l}\text { - Fortified with available material (ADA, soluble target } \\
\text { and catabolites) or concomitant drugs, as appropriate } \\
\text { - Accuracy within } 25 \%\end{array}$ \\
\hline $\begin{array}{l}\text { Matrix effect on } \\
\text { MS ionization } \\
\text { Using individual matrix lots } \\
\text { Also evaluate hemolyzed, lipemic } \\
\text { or relevant disease population } \\
\text { samples, as appropriate }\end{array}$ & NA & $\begin{array}{l}\text { - MF in six lots } \\
\text { - IS-normalized CV within 15\% } \\
\text { across lots }\end{array}$ & $\begin{array}{l}\text { - MF in 6-10 lots } \\
\text { - Compare surrogate and SIL-IS peptides in processed } \\
\text { matrix and reagent blanks } \\
\text { - IS-normalized CV within } 20 \% \text { across lots } \\
\text { - Alternatively: compare individual QC samples prepared } \\
\text { from multiple lots } \\
\text { - Accuracy (CV) within } 20 \% \text { (or } 25 \% \text { LLOQ) across lots }\end{array}$ \\
\hline Recovery & NA & $\begin{array}{l}\text { Extraction recovery should be } \\
\text { reproducible }\end{array}$ & $\begin{array}{l}\text { Overall recovery including digestion should be } \\
\text { reproducible } \\
\text { Recoveries for individual steps may be evaluated for } \\
\text { troubleshooting }\end{array}$ \\
\hline Matrix stability & $\begin{array}{l}\text { Within } 20 \% \text { of nominal } \\
\text { Determine at each storage } \\
\text { temperature }\end{array}$ & $\begin{array}{l}\text { Within } 15 \% \text { of nominal } \\
\text { temperature bracketing } \\
\text { approach may be used }\end{array}$ & $\begin{array}{l}\text { Within } 20 \% \text { of nominal } \\
\text { Determine at each storage temperature }\end{array}$ \\
\hline $\begin{array}{l}\text { Processed sample extract storage } \\
\text { stability } \\
\text { Determine at intended storage } \\
\text { temperature }\end{array}$ & NA & $\begin{array}{l}\text { - Within } 15 \% \text { of nominal } \\
\text { - Stored QC extracts measured } \\
\text { against freshly prepared curve }\end{array}$ & $\begin{array}{l}\text { - Within } 20 \% \text { of nominal } \\
\text { - Stored QC extracts measured against freshly processed } \\
\text { curve, or original curve when justified }\end{array}$ \\
\hline $\begin{array}{l}\text { Stock and working solution } \\
\text { stability }\end{array}$ & $\begin{array}{l}\text { May not be required if covered } \\
\text { by COA }\end{array}$ & $\begin{array}{l}\text { - Compare old vs freshly } \\
\text { prepared solutions } \\
\text { - Mean values within } 5-7 \% \\
\text { typical }\end{array}$ & $\begin{array}{l}\text { - Compare old vs freshly prepared solutions } \\
\text { - Protein must be digested; mean values within } 10 \% \\
\text { recommended }\end{array}$ \\
\hline Run size & NA & Validate maximum anticipated & Validate maximum anticipated \\
\hline $\begin{array}{l}\text { Carryover } \\
\text { (Blank following a ULOQ sample) }\end{array}$ & $\begin{array}{l}\text { - Generally, NA, some assay } \\
\text { technologies may need to assess } \\
\text { (e.g., Gyros, MSD and others) } \\
\text { minimize and mitigate }\end{array}$ & $\begin{array}{l}\text { - Prefer }<20 \% \text { LLOQ response } \\
\text { - Minimize and mitigate }\end{array}$ & $\begin{array}{l}\text { - Prefer }<20 \% \text { LLOQ response, may need to accept } \\
\text { higher with justification } \\
\text { - Minimize and mitigate }\end{array}$ \\
\hline Change in critical assay reagents & May need revalidation & NA & $\begin{array}{l}\text { - Primarily a concern for protein reagents } \\
\text { - Confirm by acceptable accuracy and precision in at } \\
\text { least one run; more extensive testing sometimes needed }\end{array}$ \\
\hline Critical assay reagent stability & $\begin{array}{l}\text { Appropriate testing /stability } \\
\text { programs for LBA reagents may } \\
\text { be required }\end{array}$ & NA & $\begin{array}{l}\text { Stability is demonstrated by prevalidation testing and } \\
\text { acceptability of validation and analytical runs; longer } \\
\text { term testing sometimes needed }\end{array}$ \\
\hline \multicolumn{4}{|c|}{$\begin{array}{l}\text { ADA: Antidrug antibody; COA: Certificate of analysis; IS: Internal standard; LBA: Ligand-binding assay; MF: Matrix factor; MSD: Meso Scale Discovery; NA: Not applicable; QC: } \\
\text { Quality control; RE: Relative error; SIL: Stable isotope label. } \\
\text { Reproduced with permission from [4] @ Springer Nature (2014). }\end{array}$} \\
\hline
\end{tabular}


between LC-MS, hybrid LC-MS and LBA during preclinical and clinical studies. In cases where there is an assay change within a study, the recent FDA guidance provides clear directions on bridging from one assay type to another "When a new platform is used in the development of a drug within a study, the data it produce should be bridged to that of the other method. This is best accomplished by assessing the output of both methods with a set of incurred samples."

As set forth in guidance documents, the recommendations can be modified with justification, depending on the specific type of bioanalytical method. However, regulators have recommended that proposed bioanalytical strategies be discussed with them early on, so they can understand the methodology and ask questions ahead of time [38]. Questions about peptide selection, reliability of the signature peptide in human studies, type of mass analyzer used, stability at the protein versus the peptide level, the number of peptide fragments to monitor and protein digestion have all been raised as possible 'new' steps in the process that could impact reliability of the assay [38]. Some LC-MS assay validation data for therapeutic mAbs have been shown to meet small molecule acceptance criteria. However, this is an evolving area, and wider acceptance criteria may be warranted depending on assay implementation, such as potential assay interference in the LBA step of hybrid LC-MS. Based on the collective hybrid LC-MS experience of this IQ Working Group, we endorse the 20/25 acceptance criteria [4].

Given the lack of explicit regulatory guidance and the wide use of CROs, there is also a need for ongoing communication and education on the use of these new assay formats. CROs and their sponsors need to be proactive in making regulators aware of current practices and developments in new assay approaches. Sponsors need to be diligent in documenting new approaches, and modifying various methodological and acceptance criteria in order to adopt new assay formats for the development of biotherapeutics. Inspectors may not be aware of current white papers and literature when conducting inspections, so pre-emptively providing information to regulators is recommended. In addition, standard operating procedures should reflect current approaches and any deviations must be documented with scientific justification and reference to current white papers and related literature.

\section{Future directions}

As the use of hybrid IA LC-MS and LC-HRMS increases for both routine and specialized PK and biotransformation studies, further opportunities will arise for utilization of both LBA and LC-MS-based approaches for the most comprehensive data for in-life sample sets. As drug modalities continue to become increasingly complex, collaboration and training of personnel will be important for fully integrating LBA and LC-MS approaches to generate state-of-the-art data. Within a company, this will also involve making project teams aware of analytical capabilities in-house (or available as outsourced) such that bioanalytical groups can influence hypotheses generation and plan bioanalysis in a fully integrated and seamless manner.

The need to monitor ADC DARs, fusion protein biotransformation and other biotherapeutic mass variants from in-life studies has been a driver for recent development of LC-MS methods focused on intact or subunit-level mass detection. Traditional LC-MS methods based on tryptic peptide digestion are routinely implemented, however, for drug quantification purposes, one or few peptides are typically utilized and information about the whole protein is lost in the digestion step. ADC conjugation measurements based on intact masses have been utilized for relative quantification of DAR and other biotransformation species [39,40]. DAR and ADC conjugate information can be obtained at the antibody subunit level as well $[41,42]$. Fusion proteins can be susceptible to catabolism at specific amino acid sites, and MS can provide specific clipping information from in-life studies not possible by LBA [43,44].

Relative quantification within sample for DAR or other biotransformation events can complement LBA methods, which typically rely on absolute quantification of a total (or free) antibody but are not able to measure DAR or other biotransformation events. The need to support mass variant monitoring in early discovery in-life studies has driven absolute quantification of biotherapeutic masses. Intact mAb quantification workflows based on HRMS have emerged, and intact protein PK can be monitored along with biotransformation [45,46]. Biotherapeutic mAb subunit quantification has also been shown [47]. A recent article demonstrated absolute quantification based on individual ADC DAR species at the intact level using LC-HRMS quantification of intact trastuzumb emtansine in rat plasma [48].

While the current application of HRMS is limited by sensitivity, a combination of IA capture and improved micro/nano-LC separation may spearhead intact protein quantification effort by HRMS. Additional momentum in the sensitivity and specificity of intact protein/biotransformation analysis could be achieved through parallel reaction monitoring where a precursor ion isolated in a quadrupole analyzer can be fragmented in a higher energy 
collisional dissociation cell followed by the detection of fragment ions in the orbitrap mass analyzer. Recently, using parallel reaction monitoring strategy, LOD in intact protein analysis is obtained at sub fmole level [49].

Moving forward, challenges for an integrated LBA and LC-MS approach will be the effective usage of multiple quantitative datasets and incorporation of new types of data such as HRMS intact protein and peptide biotransformation data into regulated study packages. Such integrated and new types of data may provide additional insights for understanding the safety and efficacy of large molecule biotherapeutics. Bioanalytical groups should consider the 'how and when' of complementary approaches to answer specific questions regarding PK and metabolism, and properly communicate the integrated sample results within their organizations. It may be helpful to have an ongoing dialog with regulators, as early as possible, given the increasing complexity of drug modalities and the complex bioanalytical strategies using integrated LBA, LC-MS and hybrid IA LC-MS approaches.

\section{Executive summary}

- Increasingly diverse large molecule biotherapeutic modalities have driven the need for complex bioanalytical strategies involving both ligand-binding assays (LBA) and hybrid immunoaffinity LC-MS platforms.

- The scientific expertise in LBA and LC-MS typically resides in different functions within the biopharma industry and organizational structures vary widely.

- Developing integrated approaches for the bioanalysis and biotransformation assessment of large molecules has presented both technical and operational challenges.

- Encouragingly, over time, the industry has recognized the complementary value of LBA and LC-MS and the value of integrated strategies. This has not been an easy transition.

- This IQ consortium paper presents the current perspectives across the biopharma industry; major considerations and recommendations for fully integrated strategies are discussed.

\section{Acknowledgments}

The following members of the International Consortium for Innovation and Quality in Pharmaceutical Development (IQ) Large Molecule LC-MS Working Group are acknowledged for useful discussions and review of this manuscript; M Berna, N Chemuturi, L-Z Chen, S Couffin, D Ghosh, S Hengel, T Hetzel, C Krantz, H Li, Z Liang, M Myers, H Neubert, O Pasquier, J Pav, L Staelens and F Vazvaei

The IQ Consortium leadership is acknowledged for review of this document: CM Hop, S Tse, M Gnoth and M Hall. Editorial support was provided by Anshin Biosolutions Corp.

Financial \& competing interests disclosure

The authors are employees of Genentech, a member of the Roche Group; Merck \& Co., Inc.; Novartis; UCB; GSK and BMS. The authors have no other relevant affiliations or financial involvement with any organization or entity with a financial interest in or financial conflict with the subject matter or materials discussed in the manuscript apart from those disclosed.

No writing assistance was utilized in the production of this manuscript.

\section{References}

Papers of special note have been highlighted as: $\bullet$ of interest; $\bullet \bullet$ of considerable interest

1. Neubert H, Olah T, Lee A et al. 2018 White Paper on Recent Issues in Bioanalysis: focus on immunogenicity assays by hybrid LBA/LCMS and regulatory feedback (Part 2 - PK, PD \& ADA assays by hybrid LBA/LCMS \& regulatory agencies' inputs on bioanalysis, biomarkers and immunogenicity). Bioanalysis 10(23), 1897-1917 (2018).

2. Kaur S, Pilluta R, Yu H et al. 2019 White Paper on Recent Issues in Bioanalysis: mass spectrometric method development strategies for large molecule bioanalysis. Bioanalysis 11(22), 2029-2048 (2019).

- Summary of large molecule LC-MS bioanalysis discussions from an expert group convened at a recent industry workshop.

3. Ezan E, Bitsch F. Critical comparison of MS and immunoassays for the bioanalysis of therapeutic antibodies. Bioanalysis 1(8), 1375-1388 (2009).

4. Jenkins R, Duggan JX, Aubry AF et al. Recommendations for validation of LC-MS/MS bioanalytical methods for protein biotherapeutics. AAPS J. 17(1), 1-16 (2015).

-• Recommendations in this paper are widely followed for the validation of hybrid immunoaffinity LC-MS/MS assays.

5. Zhang M, An B, Qu Y et al. Sensitive, high-throughput, and robust trapping-micro-LC-MS strategy for the quantification of biomarkers and antibody biotherapeutics. Anal. Chem. 90(3), 1870-1880 (2018). 
6. Liu L, Xu K, Li J et al. Optimizing hybrid LC-MS/MS binding conditions is critical: impact of biotransformation on quantification of trastuzumab. Bioanalysis 10(22), 1819-1831 (2018).

7. Xu K, Liu L, Maia M et al. A multiplexed hybrid LC-MS/MS pharmacokinetic assay to measure two co-administered monoclonal antibodies in a clinical study. Bioanalysis 6(13), 1781-1794 (2014).

8. Chen L RD, Philip E, Pagels S. Sequential immunoaffinity-LC/MS assay for quantitation of a therapeutic protein in monkey plasma. J. Appl. Bioanal. 3(5), 127-138 (2017).

9. An B, Zhang M, Pu J et al. High-throughput, sensitive LC-MS quantification of biotherapeutics and biomarkers using antibody-free, peptide-level, multiple-mechanism enrichment via strategic regulation of $\mathrm{pH}$ and ionic and solvent strengths. Anal. Chem. 91(5), 3475-3483 (2019).

10. Kaur S, Xu K, Saad OM, Dere RC, Carrasco-Triguero M. Bioanalytical assay strategies for the development of antibody-drug conjugate biotherapeutics. Bioanalysis 5(2), 201-226 (2013).

11. Faria M, Peay M, Lam B et al. Multiplex LC-MS/MS assays for clinical bioanalysis of MEDI4276, an antibody-drug conjugate of tubulysin analogue attached via cleavable linker to a biparatopic humanized antibody against HER-2. Antibodies 8(1), 11 (2019).

12. Xu L, Packer LE, Li C, Abdul-Hadi K, Veiby P. A generic approach for simultaneous measurements of total antibody and cleavable antibody-conjugated drug by LC/MS/MS. Anal. Biochem. 537, 33-36 (2017).

13. Kaur S, Liu L, Cortes DF et al. Validation of a biotherapeutic immunoaffinity-LC-MS/MS assay in monkey serum: 'plug-and-play' across seven molecules. Bioanalysis 8(15), 1565-1577 (2016).

- Validation data for seven hybrid LC-MS/MS assays are presented.

14. Anderson NL, Anderson NG, Haines LR, Hardie DB, Olafson RW, Pearson TW. Mass spectrometric quantitation of peptides and proteins using Stable Isotope Standards and Capture by Anti-Peptide Antibodies (SISCAPA). J. Proteome Res. 3(2), 235-244 (2004).

15. Whiteaker JR, Zhao L, Zhang HY et al. Antibody-based enrichment of peptides on magnetic beads for mass-spectrometry-based quantification of serum biomarkers. Anal. Biochem. 362(1), 44-54 (2007).

16. Sucharski FK, Meier S, Miess C et al. Development of an automated, interference-free, 2D-LC-MS/MS assay for quantification of a therapeutic mAb in human sera. Bioanalysis 10(13), 1023-1037 (2018).

17. Vasicek LA, Spellman DS, Hsieh S et al. Quantitation of a therapeutic antibody in serum using intact sequential affinity capture, trypsin digestion, and LC-MS/MS. Anal. Chem. 90(1), 866-871 (2018).

18. $\mathrm{He} \mathrm{J}, \mathrm{Su} \mathrm{D}, \mathrm{Ng} \mathrm{C}$ et al. High-resolution accurate-mass mass spectrometry enabling in-depth characterization of in vivo biotransformations for intact antibody-drug conjugates. Anal. Chem. 89(10), 5476-5483 (2017).

19. Kellie JF, Karlinsey MZ. Review of approaches and examples for monitoring biotransformation in protein and peptide therapeutics by MS. Bioanalysis 10(22), 1877-1890 (2018).

20. Bults P, Bischoff R, Bakker H, Gietema JA, Van De Merbel NC. LC-MS/MS-based monitoring of in vivo protein biotransformation: quantitative determination of trastuzumab and its deamidation products in human plasma. Anal. Chem. 88(3), 1871-1877 (2016).

21. Baillie TA. Metabolism and toxicity of drugs. Two decades of progress in industrial drug metabolism. Chem. Res. Toxicol. 21(1), 129-137 (2008).

22. Zhu M, Zhang H, Humphreys WG. Drug metabolite profiling and identification by high-resolution mass spectrometry. J. Biol. Chem. 286(29), 25419-25425 (2011).

23. Xu K, Liu L, Dere R et al. Characterization of the drug-to-antibody ratio distribution for antibody-drug conjugates in plasma/serum. Bioanalysis 5(9), 1057-1071 (2013).

24. Shen $\mathrm{BQ}, \mathrm{Xu} \mathrm{K}$, Liu L et al. Conjugation site modulates the in vivo stability and therapeutic activity of antibody-drug conjugates. Nat. Biotechnol. 30(2), 184-189 (2012).

25. Hall MP, Gegg C, Walker K et al. Ligand-binding mass spectrometry to study biotransformation of fusion protein drugs and guide immunoassay development: strategic approach and application to peptibodies targeting the thrombopoietin receptor. AAPS J. 12(4), 576-585 (2010).

26. Su D, Ng C, Khosraviani $\mathrm{M}$ et al. Custom-designed affinity capture LC-MS F(ab')2 assay for biotransformation assessment of site-specific antibody drug conjugates. Anal. Chem. 88(23), 11340-11346 (2016).

27. Liu YD, Van Enk JZ, Flynn GC. Human antibody Fc deamidation in vivo. Biologicals 37(5), 313-322 (2009).

28. Craddock A, Nadarajah S. Future trends in outsourcing: a summary of the Bioanalysis Zone Survey. Bioanalysis 9(15), 1127-1129 (2017).

- Industry survey discusses trends in outsourcing large-molecule assays.

29. Spooner N, Anderson M, Dillen L et al. The changing world of bioanalysis: summary of panel discussions. Bioanalysis 9(15), 1175-1179 (2017).

30. Abberley L. Outsourcing of bioanalysis at GSK: a hybrid approach with a robust support model. Bioanalysis 9(15), 1139-1144 (2017).

31. Hayes R. Bioanalytical outsourcing: transitioning from Pharma to CRO. Bioanalysis 9(15), 1149-1152 (2017). 
32. Guideline on Bioanalytical Method Validation. European Medicines Agency, London, UK (2011),

33. Guideline on Bioanalytical Method Validation in Pharmaceutical Development. Ministry of Health, Labour and Welfare, Tokyo, Japan (2013).

34. Resolution RDC No. 27 OF 17 May 2012. Brazilian Health Regulatory Agency (ANVISA), Brasilia, Brazil (2012).

35. Guidance for Industry Bioanalytical Method Validation. US Department of Health and Human Services, US FDA, Rockville, MD, USA (2001).

36. Guidance for Industry Bioanalytical Method Validation. US Department of Health and Human Services, US FDA, Rockville, MD, USA (2018).

37. Duggan JX, Vazvaei F, Jenkins R. Bioanalytical method validation considerations for LC-MS/MS assays of therapeutic proteins. Bioanalysis 7(11), 1389-1395 (2015).

38. Booth BP, Furmanski B. Hybrid assays: the next big thing? Bioanalysis 10(13), 975-977 (2018).

- A regulatory perspective on hybrid LC-MS assays.

39. He J, Su D, Ng C et al. High-resolution accurate-mass mass spectrometry enabling in-depth characterization of in vivo biotransformations for intact antibody-drug conjugates. Anal. Chem. 89(10), 5476-5483 (2017).

40. Li KS, Chu PY, Fourie-O'donohue A et al. Automated on-tip affinity capture coupled with mass spectrometry to characterize intact antibody-drug conjugates from blood. J. Am. Soc. Mass Spectrometr. 1-6 (2018).

41. Davis JA, Kagan M, Read J, Walles M, Hatsis P. Immunoprecipitation middle-up LC-MS for in vivo drug-to-antibody ratio determination for antibody-drug conjugates. Bioanalysis 9(20), 1535-1549 (2017).

42. Su D, Ng C, Khosraviani M et al. Custom-designed affinity capture LC-MS F (ab') 2 assay for biotransformation assessment of site-specific antibody drug conjugates. Anal. Chem. 88(23), 11340-11346 (2016).

43. Han M, Pearson JT, Wang Y et al. Immunoaffinity capture coupled with capillary electrophoresis-mass spectrometry to study therapeutic protein stability in vivo. Anal. Biochem. 539, 118-126 (2017).

44. Kang L, Camacho RC, Li W et al. Simultaneous catabolite identification and quantitation of large therapeutic protein at the intact level by immunoaffinity capture liquid chromatography-high-resolution mass spectrometry. Anal. Chem. 89(11), 6065-6075 (2017).

45. Jian W, Kang L, Burton L, Weng N. A workflow for absolute quantitation of large therapeutic proteins in biological samples at intact level using LC-HRMS. Bioanalysis 8(16), 1679-1691 (2016).

46. Lanshoeft C, CianféRani S, Heudi O. Generic hybrid ligand binding assay liquid chromatography high-resolution mass spectrometry-based workflow for multiplexed human immunoglobulin G1 quantification at the intact protein level: application to preclinical pharmacokinetic studies. Anal. Chem. 89(4), 2628-2635 (2017).

47. Kellie JF, Kehler JR, Mencken TJ, Snell RJ, Hottenstein CS. A whole-molecule immunocapture LC-MS approach for the in vivo quantitation of biotherapeutics. Bioanalysis 8(20), 2103-2114 (2016).

48. Jin W, Burton L, Moore I. LC-HRMS quantitation of intact antibody drug conjugate trastuzumab emtansine from rat plasma. Bioanalysis 10(11), 851-862 (2018).

49. Chen Y, Mao P, Wang D. Quantitation of intact proteins in human plasma using top-down parallel reaction monitoring-M. Anal. Chem. 90(18), 10650-10653 (2018). 